\title{
A Novel Approach to Select an Appropriate Requirements Prioritization Technique
}

\author{
Punam Bajaj \\ Chandigarh Engineering College, Landran
}

\author{
Vineet Arora \\ Chandigarh Engineering College, Landran
}

\begin{abstract}
Requirements prioritization has been recognized as a critical and essential but challenging activity for product development. The pressure on time-to-market and being able to plan for successive release of the software product has posed many challenges to the software engineering process. Budgetary restrictions and time-tomarket deadline often compel stakeholders to cautiously prioritize requirements. Various requirements prioritization methods are available in literature, but generally practices in companies are found to be informal. This paper evaluates various requirements prioritization methods with different characteristics and proposes a novel technique to select an appropriate method for the application. The aim of our work is to offer a method to guide the engineer through various characteristics such as ease to use, size of project, number of decisions etc. for an application. This approach is illustrated with a case study of the Travel Management Planning Website.
\end{abstract}

\section{General Terms}

Algorithms, Requirements, Management, Performance

\section{Keywords}

Prioritization Methods, Fuzzy Analytic Hierarchy Process (FAHP), Criteria, Travel Management Planning (TMP)

\section{INTRODUCTION}

$\mathrm{RE}$ is a structured process of elicitating, defining, negotiating, prioritizing and validating requirements of a system [1]. Requirements Prioritization is one of the most important activities of requirements engineering that is concerned with selecting the most important requirements out of an abundant accumulated list of all significant or insignificant requirements. Requirements Prioritization aims to select the 'right' requirements from the set of candidate requirements so that all the different key interests, technical constraints and preferences of the stakeholders are fulfilled. By defining and addressing high priority requirement before the low priority ones, one can significantly reduce the project cost and duration [2]. It is usually impossible to implement all of the requirements due to limited resources in terms of budget, staff and schedule. Therefore, it becomes mandatory for project managers to prioritize the requirements.

There are various approaches recognized by researchers for requirement prioritization $[3,4,5,6,9]$. Bubble sort technique was first introduced by Karlsson to the requirements prioritization area for ranking requirements. Bubble sort is a simple sorting algorithm that works by repeatedly stepping through the list to be sorted, comparing each pair of adjacent items and swapping them if they are in the wrong order [3]. The Priority Assessment method introduced by Kunia for assesses the priority of requirements subject to the multiple perspectives. Priority Assessment method using relationship matrix contemplates multiple perspectives of stakeholders that utilize the concept of correlation to compute weighted priorities of requirements [9]. Analytical Hierarchy Process (AHP) was introduced by Satty to the requirements area for organizing and analyzing complex decisions. AHP is a multicriteria decision-making technique that uses a pair wise comparison matrix to compute the relative value of requirements with respect to one another [4]. Fuzzy AHP (FAHP) is a systematic decision making method which includes both qualitative and quantitative technique to remove incompleteness, uncertainty and vague data [5]. Requirements Triage uses cluster based automated method expresses various concerns of stakeholders to multiple categories of requirements such as feature based, non-functional requirements and other cluster requirements [6]. The requirements prioritization techniques have been used in various applications viz. supplier chain management [7], for enhancing the business performance, in Core Banking transformation programs [8], web browser system, telephone network, GPS technology system, and social networking system.

Concerning with the issues associated with requirements prioritization, this paper evaluates various prioritization methods available in the literature and proposes a novel approach to select an appropriate prioritization method for a specific application. It is observed that none of the prioritization methods may be absolutely perfect to meet all the desired criteria of an application. A prioritization method for one application may not be suited to another kind of application. A wrong selection of the prioritization method may result in enhanced development cost and delivery time causing customers dissatisfaction [16]. This paper aims to select the 'right' method from the set of candidate prioritization methods available in literature so that all the different criteria desired for a specific application are fulfilled with in the technical constraints. This paper first evaluates various prioritization methods with different characteristics and proposes a novel technique to identify an appropriate method for a specific application that is bounded by a number of criteria.

The organization of the paper is as follows: Section 2 provides an overview of various prioritization methods available in literature and section 3 evaluates these prioritization methods on the basis of characteristics exhibited. Section 4 presents a novel approach to identify a requirement engineering method. Section 5 presents the experimental study of the Travel Management Planning Website. And finally, section 6 concludes the paper. 


\section{OVERVIEW OF REQUIREMENTS PRIORITIZATION METHODS}

Prioritization methods assist developers to rank excessive accumulated requirements as per their importance. Various prioritization methods have been reported in literature with their associated features. The objective is to illustrate and evaluate the existing prioritization methods against a number of parameters such as technique used, multi-criteria, multiple stakeholders and complexity analysis etc. as presented in Table 1. This section incorporates the brief discussion of every prioritization method.

\subsection{Bubble Sort}

Bubble sort technique introduced by Karlsson to the requirements prioritization area for ranking requirements. It refers to as sinking sort, is a simple sorting algorithm that works by repeatedly stepping through the list to be sorted, comparing each pair of adjacent items and swapping them if they are in the wrong order. The pass through the list is repeated until no swaps are needed, which indicates that the list is sorted. The algorithm gets its name from the way smaller elements "bubble" to the top of the list. Because it only uses comparisons to operate on elements, it is a comparison sort. Although the algorithm is simple, most of the other sorting algorithms are more efficient for large lists.

As with AHP, the required numbers of pairwise comparisons in bubble sort are more but, the decision maker only has to determine which of the two requirements is of higher priority, not to what extent [3].

\subsection{AHP (Analytical Hierarchy Process)}

The Analytical Hierarchy Process is a multi-criteria decisionmaking technique that uses a pair wise comparison matrix to compute the relative value of requirements with respect to one another [4]. The basic idea of AHP is to calculate the priorities of requirements by comparing all unique pairs of requirements to estimate their relative importance. In other words, the person performing the comparison has to decide which requirement is more important, and to what extent.

It is an easier method to use due to pair wise comparison of requirements which supports various requirements to be prioritized w.r.t. multi-criteria, but it has a number of drawbacks. It works only with a single decision maker only while in real applications various stakeholders are involved to give their different opinions and it lacks incorporation of views of various stakeholders Secondly, it does not really scale well because of the high number of required pair-wise comparisons $(n \times(n-1) / 2)$. The number of pair-wise comparisons grows exponentially: we need "only" 45 comparisons with 10 requirements but with five times more requirements the number of comparisons is as much as 1225 .

\subsection{Fuzzy AHP}

Zadeh first introduced the fuzzy set theory. Fuzzy set theory generalizes classical sets in an attempt to model and simulate human linguistic reasoning in a domain characterized by incomplete, uncertain and vague data. Fuzzy AHP termed as extended form of AHP, also works on the conventional concept of pair wise comparison of requirements except that it captures the vagueness and fuzziness inherent in human thoughts by placing fuzzy linguistic terms on a scale of triangular fuzzy numbers to improve the conventional scaling scheme [5]. Stakeholders are not being able to compare every pair of requirements exactly in crisp terms.
Defuzzification is the process of producing a quantifiable result for the values which are approximate rather than precise, given fuzzy sets and corresponding membership degrees.

The Fuzzy AHP inherits all features of AHP such as simplicity of computation, having less computational expense, lack of mathematical operation but it incorporates the same drawback i.e. large number of comparisons of requirements initially made by decision maker[14].

\subsection{Priority Assessment}

Kunia introduces Priority Assessment method which assesses the priority of requirements subject to the multiple perspectives [9]. A relationship matrix is used to analyze the impact between requirements and facilitate the integration process which assesses their priorities based on their relationships from multiple perspectives. It allows the development team to resolve conflicts effectively and concentrate their valuable time and resources on the critical few requirements from multiple perspectives that directly contribute to high customer satisfaction. Factors concerning different stakeholders such as business value, risks, relation to other requirements etc., should be considered while prioritizing requirements. This method initially integrates the requirements captured from 2 stakeholders, then integrated set of requirements are integrated again with the requirements of $3 \mathrm{rd}$ stakeholder and likewise integrated set of requirements resulted from (s-1) stakeholders are amalgamated with the requirements of nth stakeholder and hence reaches to final consensual requirements having their own priority [10].

\subsection{Requirements Triage}

Requirements Triage process has been named as Pirogov that encompasses three stages to obtain a prioritized list of requirements [6]. Firstly, requirements captured from a large set of stakeholders are organized into a number of clusters of various features using various clustering methods. Secondly, so obtained clusters are prioritized manually by human analysts using either of prioritization methods such as AHP or B-Tree reported in literature. Various stakeholders can be negotiated at the cluster levels. Thirdly, requirements among clusters are prioritized by computing prioritization score and global priority score.

\section{EVALUATING REQUIREMENTS PRIORITIZATION METHODS}

The various prioritization methods are reported in the literature but the application of one method for a specific application may be overkill for another application. This necessitates that prioritization method should be evaluated on the number of characteristics such as Concept, Ease of Use, Fuzziness, Multi-criteria, Multi-person, Speed and Complexity analysis. These characteristics are discussed below in brief.

a) Concept:It is the systematic and analysis of the methods, rules, and postulates employed by the prioritization method.

b) Ease of Use: It is used to describe the characteristic that a user can easily use and implement the prioritization method.

c) Size of project: It measures the number of functional requirements supported by the prioritization method without degrading the performance of the system.

d) Fuzziness: It refers to the vagueness of human thought associated with the recommendations of experts or stakeholders [12]. 
e) Multi-criteria: It refers to the multiple attributes associated with a prioritization method [11].

f) Multi-person: It refers to the multiple stakeholders involved with the application. g) Speed: It refers to how fast, the prioritization method executes the given prioritization method.

h) Complexity: The number of comparisons required to execute the prioritization method [13].

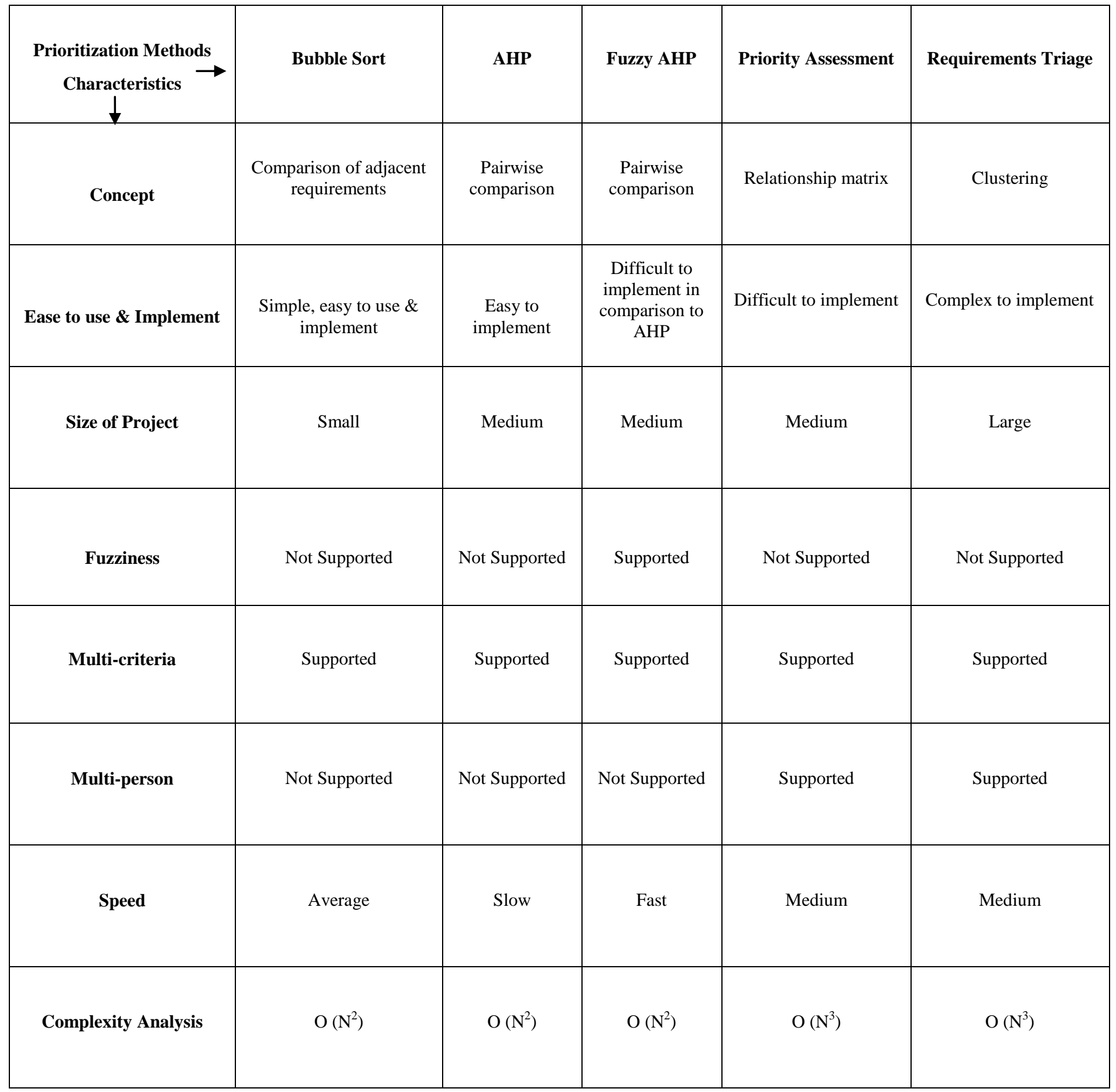

Table1. Subjective Evaluation of various Prioritization Methods 
Based on the empirical evidence, it is quite impossible to say, which of the method is the best one. It really depends on the situation. For example, if you don't need to know the relative differences between requirements, using a ratio scale technique can be overkill and more simple methods, such as Bubble Sort might be enough [15]. But, for large projects, Requirement Triage seems suitable for requirements prioritization. Table 1 lists the characteristics of various prioritization methods using linguistic terms.

\section{SELECTION OF AN APPROPRIATE PRIORITIZATION METHOD}

The selection of an appropriate prioritization method from a given set of various prioritization methods $\left\{\mathrm{M}_{1}, \mathrm{M}_{2}, \ldots . \mathrm{M}_{\mathrm{n}}\right\}$ is guided by comparing their profile with distinct characteristics $\left\{C_{1}, C_{2}, \ldots C_{n}\right.$ \}against a desired profile of a specific software application in terms of given criteria.

Algorithm to select appropriate prioritization method is given as:

(1) Convert fuzzy values specified in Table 1 to its corresponding crisp values using defuzzification method [10]. This matrix of all crisp values can be termed as:

$$
R=\left[r_{i j}\right] \text { i.e }
$$

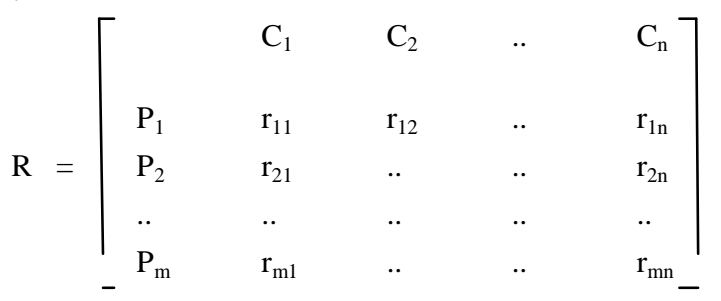

where all entries of this matrix $\mathrm{R}$ expresses degree to which characteristic $C_{j}$ is satisfied by method $P_{i}(i=1 . . m)$ and $(j=1 . . n)$.

(2) Normalize the values of matrix $R$ by dividing each value of this matrix by the maximum value of the matrix. All entries of this matrix are real numbers in $[0,1]$.

$$
\mathrm{n}=\left(\frac{\mathrm{r}}{\max }\right)
$$

where max is the maximum value of the matrix

(3) Identify an application for which a suitable method is desired. This application is bounded by a number of criteria. Assign the appropriate weight that ranges from 0 (not important or applicable) to 5 (absolutely essential) to these criteria.

$$
\mathrm{W} \quad\left[\begin{array}{l}
\mathrm{w}_{1} \\
\mathrm{w}_{2} \\
. . \\
\mathrm{w}_{\mathrm{n}}
\end{array}\right]
$$

Normalize the values of matrix $W$ as done in step 2.
(4) Multiply the matrix $R$ with Weight matrix $W$ to obtain matrix $S$. The value of the resultant matrix $S$ will be used to select the method that is most appropriate for the project.

(5) Arrange the values of matrix $S$ in decreasing order to obtain list of methods in increasing order of suitability for a given application.

This algorithm assists the manager to select an appropriate method for a specific application.

\section{CASE STUDY}

In order to illustrate the proposed methodology, an experimental study of the Travel Management Planning Website was performed. The aim of the study was to select the appropriate prioritization method for the website. Five prioritization methods namely Bubble sort, AHP, FAHP, Priority Assessment and Requirement Triage and five characteristics such as Ease of use, Size of project, Fuzziness, Multi-criteria and Multi-person were selected to simplify the study. Three domain experts were requested to give their recommendation about degree to which characteristic $C_{j}$ is possessed by method $\mathrm{P}_{\mathrm{i}}$ using linguistic terms.

Defuzzification was used to convert the linguistic terms to crisp values and results are shown in matrix $R$.

$$
\mathrm{R}=\left[\begin{array}{cccccc} 
& \mathrm{C}_{1} & \mathrm{C}_{2} & \mathrm{C}_{3} & \mathrm{C}_{4} & \mathrm{C}_{5} \\
& & & & & \\
\mathrm{P}_{1} & 4 & 2 & 0 & 0 & 4 \\
\mathrm{P}_{2} & 4 & 3 & 0 & 5 & 0 \\
\mathrm{P}_{3} & 3 & 3 & 5 & 5 & 4 \\
\mathrm{P}_{4} & 3 & 3 & 0 & 5 & 2
\end{array}\right]
$$

The matrix $R_{n}$ was obtained after normalizing the values of matrix R.

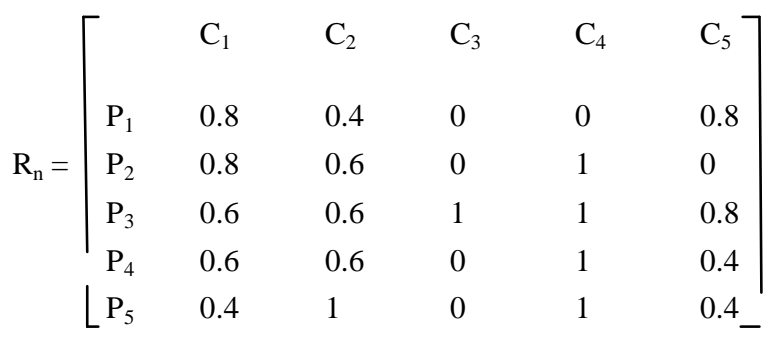

Three stakeholders including Project Manager, System Engineer \& Website Maintainer were requested to give their requirements for the website in the form of desired criteria. These criteria were assigned weights in the scale of 0 to 5 to obtain the matrix W.

$$
\mathrm{W}=\left[\begin{array}{l}
3 \\
3 \\
2 \\
4 \\
5
\end{array}\right]
$$

The matrix $\mathrm{W}_{\mathrm{n}}$ was obtained by normalizing the matrix $\mathrm{W}$. 


$$
\mathrm{W}_{\mathrm{n}}=\left[\begin{array}{l}
0.6 \\
0.6 \\
0.4 \\
0.8 \\
1
\end{array}\right]
$$

$\mathbf{R}_{\mathbf{n}}$ is an $n \times m$ matrix and $\mathbf{W}_{\mathbf{n}}$ is an $m \times p$ matrix, the result $\mathbf{S}$ of their multiplication is an $n \times p$ matrix.

$$
\mathrm{S}=\left[\begin{array}{l}
1.52 \\
1.64 \\
2.72 \\
1.92 \\
2.04
\end{array}\right]
$$

The matrix $S_{n}$ was obtained by arranging the values of matrix $S$ in decreasing order to obtain the methods in the increasing order of their suitability for the website. The results of the study are presented in Table 2 and illustrated in Figure 1. It shows that Fuzzy AHP is found most suitable followed by Requirement Triage, Priority Assessment and AHP. Bubble Sort was found least suitable for the website.

$$
\mathrm{S}_{\mathrm{n}} \quad=\left[\begin{array}{l}
2.72 \\
2.04 \\
1.92 \\
1.64 \\
1.52
\end{array}\right]
$$

Table 2: Prioritized Methods in Ascending Order of their suitability

\begin{tabular}{|l|l|l|l|l|l|}
\hline Methods & FAHP & $\begin{array}{l}\text { Requirement } \\
\text { Triage }\end{array}$ & $\begin{array}{l}\text { Priority } \\
\text { Assessment }\end{array}$ & AHP & $\begin{array}{l}\text { Bubble } \\
\text { Sort }\end{array}$ \\
\hline Values & 2.72 & 2.04 & 1.92 & 1.64 & 1.52 \\
\hline
\end{tabular}

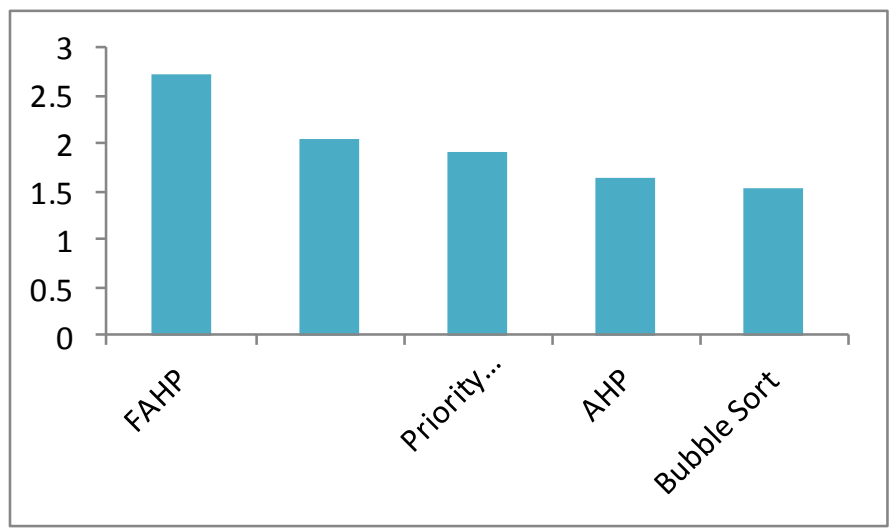

Fig.1. A Comparative Analysis of Various Prioritization Methods

\section{CONCLUSIONS}

Requirements Prioritization aims to select the 'right' requirements from the set of candidate requirements so that all the different key interests, technical constraints and preferences of all stakeholders are fulfilled. This paper evaluates the various prioritization techniques available in literature and evaluates them on the basis of various characteristics possessed by them. These characteristics assist the manager to identify an appropriate method for a specific application which may be bounded by a number of desired criteria. These criteria are assigned weights by the stakeholders based on the nature of project. Finally, an algorithm is presented to identify a method that matches closely with the desired criteria specified for an application. This algorithm may assist the manager to achieve customers satisfaction as wrong selection of a prioritization method may result in enhanced development cost and delivery time.

\section{REFERENCES}

[1] Nuseibeh, B., \& Easterbrook, S. Requirements Engineering, "A Roadmap", Paper presented at the the 22nd International conference on Software Engineering (ICSE 2000), Limerick, Ireland.

[2] Vibha Gaur, Anuja Soni, "An Integrated Approach to Prioritize Requirements Using Fuzzy Decision Making", IACSIT International Journal of Engineering and Technology, Vol.2, No.4, August 2010 ISSN: 1793-8236, pp. 320-328.

[3] J. Karlsson, C. Wohlin, and B. Regnell, "An evaluation of methods for prioritizing software requirements," Information and Software Technology,vol. 39, no. 14-15, pp. 939 - 947, 1998.

[4] T.L. Saaty, "The Analytic Hierarchy Process", McGraw-Hill, Inc. (1980).

[5] Vibha Gaur, Anuja Soni, "Evaluating Degree of Dependency from Domain Knowledge using Fuzzy Inference System", Springer in CCIS Series, 204, pp. 101-111, 2011 CCSEIT2011.

[6] Laurent, P., "Towards Automated Requirements Triage", 2007, IEEE, ISBN: 978-0-7695-2935-6,pp.131-140

[7] Sreekumar and S. S. Mahapatra, "A fuzzy multi-criteria decision making approach for supplier selection in supply chain management", African Journal of Business Management Vol.3 (4), pp. 168-177, April, 2009.

[8] S.S.Jahagirdar, M. Kumar, "Application of Value Based Requirement Prioritization in a Banking Product Implementation", 2012, ICSEM, ISSN: 978-1-4673-5729-6, pp. 157-161.

[9] Yuji Kyoya, Priority Assessment of Software Requirements from Multiple Perspective, ISBN: 0-7695-2209-2,volume:1, pp:410-415, 2004, IEEE.

[10] Vibha Gaur, Anuja Soni, S.K.Matoo, "An Application of fuzzy criteria decision making in the selection of an appropriate prioritization method" , IACSIT International Journal of Information Technology and Decision Making, 2010

[11] K. Atanassov, G. Pasi, R.Yager, "Intuitionistic fuzzy interpretations of multi-person multi-criteria decision 
making”, First International IEEE Symposium on Intelligent Systems, 2002, Volume 1, pp. 115-119.

[12] J. Karlsson, "Software requirements prioritizing", in: Proc. of 2nd IEEE International Conference on Requirements Engineering (1996) pp. 110-116.

[13] Karlsson, J., Wohlin, C., \&Regnell, B. (1998). An evaluation of methods for prioritizing software requirements. Information and Software Technology, 39(14-15), 939-947.
[14] Regnell B, Host M, Nattoch Dag J, Beremark P, HjelmT(2001),"An industrial case study on distributed prioritization in market driven requirements engineering for packaged software. Requirements Engineering 6(1):51-62.

[15] Mohammad Shabbir Hasan," An Evaluation of Software Requirement Prioritization Techniques", (IJCSIS) Vol. 8, No. 9, December 2010.

[16] I. K. Bray, "An Introduction to Requirements Engineering”, Addison Wesley, 2002. 